\section{Asma e fatores associados em adolescentes de 13 e 14 anos em São Luís, Maranhão, Brasil}

\author{
Asthma and associated factors in students 13 and \\ 14 years of age in São Luís, Maranhão State, Brazil
}

\author{
1 Universidade Federal do \\ Maranhão, São Luís, Brasil. \\ 2 Faculdade Santa Terezinha, \\ São Luís, Brasil. \\ Correspondência \\ W. L. Lima \\ Programa de Pós-graduação \\ em Saúde Materno-Infantil, \\ Universidade Federal do \\ Maranhão. \\ Praça Gonçalves Dias 21, \\ Prédio de Medicina, 2o andar, \\ São Luís, MA 65020-240, \\ Brasil. \\ willyfisio@gmail.com
}

\begin{abstract}
The aim of this study was to estimate the prevalence of asthma and associated factors among students 13 and 14 years of age in São Luís, Maranhão State, Brazil. A cross-sectional study was conducted using the questionnaire developed by ISAAC (International Study of Asthma and Allergies in Childhood) and an additional questionnaire from July 2008 to May 2009, including 3,069 adolescents. Asthma prevalence (wheezing in the previous 12 months) was 12.7\%, lifetime prevalence was 32.4\%, 3.9\% reported difficulty speaking due to wheezing, and 9.8\% reported wheezing after exercise. In the multivariate analysis, factors associated with increased asthma prevalence were family history of asthma, respiratory infection and wheezing in early life, eczema, allergic rhinitis, and passive smoking. Asthma prevalence was lower than for Brazil as a whole. Factors most strongly associated with asthma prevalence in these adolescents were family history of asthma $(P R=3.86)$, wheezing in early childhood $(P R=4.58)$, and allergic rhinitis $(P R=3.21)$.
\end{abstract}

Asthma; Adolescent; Prevalence
Willy Leite Lima 1

Elisângela Veruska Nóbrega Crispim Leite Lima 2 Maria do Rosário da Silva Ramos Costa 1

Alcione Miranda dos Santos 1

Antônio Augusto Moura da Silva 1

Erika Saiki Costa 2

\section{Introdução}

A asma é uma das doenças crônicas mais comuns entre crianças e adolescentes, gerando altos custos para o sistema de saúde, absenteísmo na escola e dos pais no trabalho, além de interferir na qualidade de vida dos indivíduos. É considerada uma afecção potencialmente grave e, quando não tratada de forma adequada, pode ser fatal 1,2 .

Mundialmente, a asma afeta 300 milhões de pessoas, com projeções de aumento em 2025 para mais 100 milhões de asmáticos 3 . Cerca de $5 \%$ a $10 \%$ da população mundial tem asma, sendo que 1/3 tem idade inferior a 18 anos. Metade de todos os casos começa a apresentar sintomas antes dos cinco anos de idade e $25 \%$ após os 40 anos. Anualmente, nos Estados Unidos, cerca de 2,2 milhões de visitas ambulatoriais são feitas para tratamento da asma em crianças 2 .

Apesar da progressão no entendimento de sua fisiopatologia e de crescentes ofertas de tratamento, estudos demonstraram aumento da prevalência, morbidade e mortalidade da asma em vários países nas últimas décadas 4,5 . Diversas teorias foram apontadas para explicar esse aumento, mas nenhuma explicação mais convincente para esse fenômeno foi ainda encontrada 6 . A interação entre fatores genéticos e ambientais tem sido relacionada ao aumento na prevalência de asma e outras doenças alérgicas, mas os fatores ambientais são provavelmente 
os maiores determinantes da manifestação dessas doenças 7. Vários estudos têm demonstrado fatores associados ao aumento na prevalência da asma, como poluição, mudanças dietéticas, exposição alergênica e melhores condições de higiene 8 .

A constatação de aumento na prevalência da asma é dificultada pela falta de uma definição aceita amplamente e pela ausência de medidas objetivas com alta sensibilidade e especificidade, aplicáveis para crianças e grandes populações 9 . Os estudos epidemiológicos devem ser realizados com questões padronizadas, avaliação de gravidade e medidas objetivas, conduzidos em mais de uma ocasião em todo o mundo para, de forma mais confiável, acompanhar a tendência mundial da prevalência de asma e detectar variações em diferentes regiões geográficas 9,10.

Nesse sentido, foi idealizado o International Study of Asthma and Allergies in Childhood (ISAAC), que permitiu avaliar a prevalência e gravidade de asma e doenças alérgicas em crianças e adolescentes em diferentes partes do mundo, empregando-se método padronizado 11,12. Tal estudo apontou que a prevalência mundial de asma entre adolescentes (13 e 14 anos) foi de 13,7\%, oscilando entre $1,5 \%$ e $32,6 \% 1$.

Estudos epidemiológicos sobre a asma no Brasil ainda são limitados, em função de sua extensão territorial, desconhecendo-se a verdadeira dimensão da doença nas diferentes regiões 13 . O estudo ISAAC foi realizado no Brasil em algumas cidades, constatando prevalência de asma maior nas cidades de Salvador (24,6\%) e Vitória da Conquista (30,5\%), Bahia, e menor em Maceió $(14,8 \%)$, Alagoas, e Itajaí (12,3\%), Santa Catarina, sendo a prevalência média de asma entre os adolescentes brasileiros próxima de $20 \% 12$.

Inquérito de base populacional, realizado no Brasil em 2003, a PNAD (Pesquisa Nacional por Amostra de Domicílios) incluiu entre seis doenças crônicas a asma. Neste inquérito foi revelado que esta afecção apresenta alta prevalência em crianças, decrescendo até a faixa situada entre os 30 e 50 anos e retorna a aumentar a partir desta idade 14 .

Neste contexto, conhecer a prevalência de asma em adolescentes em regiões não incluídas, no estudo ISAAC é de grande relevância, visto que tal conhecimento possibilitará implementação de estratégias para a promoção da saúde. O presente estudo, utilizando metodologia padronizada pelo protocolo ISAAC, teve como objetivo estimar a prevalência e a gravidade de sintomas de asma e fatores associados em escolares de 13 e 14 anos no Município de São Luís, Maranhão, Brasil.

\section{Métodos}

Trata-se de um estudo analítico, observacional, com delineamento transversal. A amostra foi constituída por escolares, adolescentes de 13-14 anos de idade, do Município de São Luís. Segundo estimativa do Instituto Brasileiro de Geografia e Estatística (IBGE. Cidades@. http://www.ibge. gov.br/cidadesat/topwindow.htm?1, acessado em 04/Abr/2009), São Luís encontrava-se com 957.515 habitantes em 2007. O clima de São Luís é tropical, quente e úmido, com temperatura oscilando entre 20 e 31 graus. Apresenta duas estações distintas: a estação seca, de agosto a dezembro, e a estação chuvosa, de janeiro a julho (Centro de Previsão de Tempo e Estudos Climáticos. http://www.cptec.inpe.br, acessado em 04/ Abr/2009).

Compõem o universo deste estudo todas as escolas localizadas na zona urbana do Município de São Luís que tinham alunos matriculados na faixa etária de 13 e 14 anos, conforme o censo escolar de 2007. De acordo com este censo, havia 330 escolas com um total de 38.428 alunos na referida faixa etária. Destas, foram excluídas todas as escolas da zona rural e as que tinham até 10 alunos, resultando um total de 261 escolas. As escolas com menos de 86 alunos foram agregadas de modo que as mesmas formassem conglomerados com mais de 86 alunos. Portanto, a população de estudo foi composta por 195 conglomerados e 35.979 alunos.

A seleção da amostra foi feita em dois estágios. No primeiro estágio, com a finalidade de selecionar as escolas, o número total de alunos na população em estudo foi dividido por 40 (número de conglomerados na amostra). Obtido o intervalo de amostragem, foram identificados os conglomerados proporcionalmente ao intervalo de amostragem de alunos. Se o conglomerado sorteado possuísse mais de uma escola, todas as escolas pertencentes a este conglomerado eram selecionadas, resultando em 48 escolas selecionadas.

No segundo estágio, para definição dos alunos nas escolas selecionadas, foi realizada amostragem proporcional ao número de alunos em cada escola. Após a obtenção da listagem com o nome dos alunos, foi realizado sorteio aleatório, para seleção dos adolescentes a serem incluídos no estudo.

Para determinação do número de alunos para compor a amostra, foi utilizado o critério adotado pelo ISAAC, o qual estabelece um número de 3 mil participantes na faixa etária entre 13 e 14 anos 15. Considerando possíveis perdas de aproximadamente $15 \%$, o tamanho da amostra foi fixado em 3.440 alunos. 
Este estudo foi aprovado pelo Comitê de Ética em Pesquisa envolvendo Seres Humanos do Hospital Universitário Presidente Dutra, Universidade Federal do Maranhão, segundo parecer $n^{\circ}$. $215 / 2007$. Após a autorização para a realização da pesquisa pelos diretores ou coordenadores das escolas, todos os pais ou responsáveis pelos alunos participantes desta pesquisa assinaram o Termo de Consentimento Livre e Esclarecido. Após seleção dos alunos, o(s) pesquisador(es) fazia(m) uma explanação sobre a pesquisa. Em seguida, os alunos recebiam os questionários e o termo de consentimento para serem apreciados pelos pais em sua residência. Quando autorizada a participação do aluno, este respondia os questionários em sua residência, os quais deveriam ser devolvidos posteriormente com termo de consentimento assinado. Houve o cuidado de não ser mencionado o termo asma, referindo-se ao estudo como um estudo sobre doenças respiratórias, conforme orientação fornecida pelo manual do ISAAC 16.

Para coleta de dados, utilizou-se o questionário padronizado do protocolo ISAAC - módulo Asma, traduzido para o português e validado por Solé et al. 17. Este questionário é constituído por oito questões sobre sintomas atuais e passados de asma, avaliando também a gravidade da doença, sendo a investigação limitada aos últimos 12 meses, com o objetivo de minimizar erros de memória e possíveis interferências sazonais. Presença de tosse seca noturna sem estar resfriado, de sibilos (chiado no peito) pós-exercícios físicos, sono perturbado e dificuldade de fala por sibilos e a frequência de sibilos nos últimos 12 meses foram avaliados como sintomas associados à asma 15 .

Ainda para a coleta de dados foi utilizado também um questionário complementar baseado em algumas questões do questionário complementar da Fase II do ISAAC 18, reformulado pelos autores desta pesquisa, que procuraram investigar alguns fatores associados à asma, adequando os termos à linguagem da população local onde este estudo foi realizado.

Neste questionário havia perguntas sobre história familiar de asma (pai e/ou mãe com diagnóstico de asma), infecção respiratória (sinais e sintomas de bronquiolite viral) nos dois primeiros anos de vida (início da vida), prematuridade, exposição passiva à fumaça de tabaco (presença de fumantes no domicílio) e exposição intradomiciliar aos pêlos de animais domésticos (presença de gato ou cachorro no domicílio), tabagismo materno durante a gestação do pesquisado, sibilância transitória precoce (sibilos pelo menos 3 vezes nos seis primeiros meses de vida), além de presenças de eczema atópico e rinite alérgica.
A distribuição e o recebimento de ambos os questionários com o Termo de Consentimento Livre e Esclarecido ocorreu no período entre julho de 2008 a maio de 2009.

Para a avaliação da prevalência de asma foi utilizada a questão sobre a presença de sibilos nos últimos 12 meses, sendo denominados asmáticos os adolescentes que responderam afirmativamente, e não asmáticos, os que apresentaram resposta negativa a essa questão.

O diagnóstico médico de asma foi considerado se o aluno respondesse de forma afirmativa que alguma vez na vida teve asma ou bronquite 13 .

Primeiramente, foi realizada a análise descritiva. Em seguida, para identificar quais os fatores estudados estão associados com a asma, foi utilizado o modelo de regressão logística simples. As variáveis cujo valor de $\mathrm{p}$ foi menor que 0.20 foram incluídas no modelo de regressão logística múltiplo. A seleção das variáveis foi realizada pelo método passo a passo (stepwise) com eliminação retrógrada de variáveis. Apenas as variáveis com valor de $\mathrm{p}$ menor do que 0.05 permaneceram no modelo final. Foram estimados os odds ratios (OR) e seus respectivos intervalos de 95\% de confiança (IC95\%). Às categorias de referência atribuiu-se OR de 1,00. Os dados foram analisados no programa estatístico Stata 10.0 (Stata Corp., College Station, Estados Unidos). A regressão logística foi escolhida, pois o OR produz estimativa não viciada da razão de densidade de incidências em estudos transversais onde o evento tem alta prevalência 19 .

\section{Resultados}

Foram distribuídos 3.440 questionários, entretanto 371 questionários continham perguntas que não foram respondidas, sendo excluídos do estudo (10,8\%). Desta forma, a amostra estudada foi composta por 3.069 alunos, dos quais 1.136 (37\%) eram do sexo masculino e 1.601 (52\%) tinham 13 anos de idade. Os alunos cursavam a sétima ou oitava série do ensino fundamental.

Afirmaram ter apresentado "sibilância alguma vez na vida" $32,4 \%$ ( $\mathrm{n}=996)$, enquanto $12,7 \%$ ( $\mathrm{n}=390)$ responderam positivamente à questão sobre "presença de sibilos nos últimos 12 meses".

A Tabela 1 apresenta os dados referentes à prevalência dos sintomas de asma como frequências de "sibilos nos últimos 12 meses" e de "sibilos impedindo o sono", "sibilo forte dificultando a fala", "sibilo após exercícios físicos" e "tosse seca noturna sem infecção", segundo o gênero. Caso o aluno respondesse que não apresentou sibilos alguma vez na vida, era orientado 
Prevalência de sintomas de asma em escolares de 13 e 14 anos, de acordo com o sexo. São Luís, Maranhão, Brasil, 2009.

\begin{tabular}{|c|c|c|c|c|c|c|c|}
\hline \multirow[t]{2}{*}{ Variáveis } & \multicolumn{3}{|c|}{ Masculino $(N=390)$} & \multicolumn{3}{|c|}{ Feminino $(\mathrm{N}=606)$} & \multirow[t]{2}{*}{ Valor de $\mathrm{p}$} \\
\hline & $\mathbf{n}$ & $\%$ & IC95\% & n & $\%$ & IC95\% & \\
\hline Frequência de sibilos nos últimos 12 meses & & & & & & & 0,940 \\
\hline Nenhuma & 216 & 61,6 & $57,0-66,9$ & 394 & 60,9 & $56,9-64,6$ & \\
\hline $1-33$ & 115 & 32,8 & $27,9-37,5$ & 221 & 34,2 & $30,6-38,0$ & \\
\hline 4-12 & 13 & 3,7 & $2,0-5,9$ & 22 & 3,4 & $2,1-5,1$ & \\
\hline$>12$ & 6 & 1,7 & $0,7-3,6$ & 9 & 1,7 & $0,8-3,0$ & \\
\hline Sibilos impedindo o sono & & & & & & & 0,110 \\
\hline Nunca & 272 & 77,7 & $73,2-81,7$ & 464 & 71,8 & $68,2-75,3$ & \\
\hline$<1 \mathrm{vez} / \mathrm{semana}$ & 46 & 13,1 & $9,9-16,8$ & 113 & 17,4 & $14,5-20,5$ & \\
\hline$\geq 1 \mathrm{vez} / \mathrm{semana}$ & 32 & 9,1 & $6,3-12,3$ & 69 & 10,6 & $8,3-13,2$ & \\
\hline Sibilos dificultando a fala & & & & & & & 0,062 \\
\hline Sim & 33 & 9,4 & $6,8-12,8$ & 87 & 13,4 & $10,9-16,3$ & \\
\hline Não & 317 & 90,5 & $87,2-93,2$ & 559 & 86,3 & $83,2-88,8$ & \\
\hline Sibilos após exercício físico & & & & & & & 0,630 \\
\hline Sim & 108 & 9,5 & $6,8-12,8$ & 194 & 10,0 & $7,8-12,6$ & \\
\hline Não & 1.028 & 90,4 & $87,2-93,2$ & 1.739 & 89,9 & $87,3-92,1$ & \\
\hline Tosse seca noturna & & & & & & & 0,001 \\
\hline $\operatorname{Sim}$ & 269 & 23,6 & $19,5-28,1$ & 560 & 28,9 & $25,5-32-1$ & \\
\hline Não & 867 & 76,3 & $71,9-80,5$ & 1.373 & 71,0 & $67,4-74,5$ & \\
\hline
\end{tabular}

IC95\%: intervalo de 95\% de confiança.

a não responder às demais questões referentes a presença de sibilos, conforme especificação no questionário ISAAC. Assim, apenas 996 responderam a todas as questões referentes aos possíveis sintomas de asma. No que se refere à frequência de sibilos, $32,8 \%$ dos meninos e $34,2 \%$ das meninas relataram ter tido de 1-3 crises de sibilos nos últimos 12 meses. Sibilos impedindo o sono menos de uma noite por semana foram referidos por $17,4 \%$ das meninas e $13,1 \%$ dos meninos, sem diferença significante entre os sexos. Meninas relataram presença de sibilos nos últimos 12 meses impedindo a fala em $13,4 \%$, enquanto meninos os referiram em $9,4 \%$. A diferença entre os sexos não foi significante. Meninas relataram mais sibilos após exercícios físicos (10\%) do que os meninos $(9,5 \%)$, mas a diferença não foi estatisticamente significante. Maior prevalência de tosse seca noturna foi observada entre meninas (28,9\%) em relação aos meninos (23,6\%), p < 0,05 (Tabela 1).

O diagnóstico médico de asma (relacionado com "asma alguma vez na vida") com o acréscimo do termo "bronquite" (utilizado em nossa região como sinônimo de asma) foi apontado por $19,1 \%(n=587)$ dos estudantes, sendo esta prevalência semelhante nos sexos masculino (19,8\%) e feminino $(18,6 \%$; $\mathrm{p}=0,407)$.
Na análise não ajustada foi observado que história familiar de asma (OR = 3,86; IC95\%: 3,01-4,96; $\mathrm{p}<0,001$ ), infecção respiratória prévia (OR = 3,60; IC95\%: 2,83-4,58; p < 0,001), sibilos no início da vida (OR = 4,58; IC95\%: 3,58-5,85; $\mathrm{p}<0,001)$, eczema (OR = 2,18; IC95\%: 1,75-2,71; $\mathrm{p}<0,001)$, exposição a tabaco (OR = 1,64; IC95\%: 1,32-2,05; $\mathrm{p}<0,001)$, nascimento pré-termo (OR = 1,59; IC95\%: 1,10-2,30; $p=0,013$ ), exposição a gato/ cachorro (OR = 1,26; IC95\%: 1,01-1,59; $\mathrm{p}=0,040)$, tabagismo materno na gravidez $(\mathrm{OR}=$ 1,52; IC95\%: 1,06-2,17; $\mathrm{p}=0,021$ ) e rinite alérgica $(\mathrm{OR}=3,21$; IC95\%: 2,55-4,04; $\mathrm{p}<0,001$ ) estiveram estatisticamente associados com a presença da asma (Tabela 2).

Após o ajuste do modelo logístico, permaneceram associados à presença de asma: exposição passiva à fumaça de tabaco $(\mathrm{OR}=1,41)$, história familiar de asma $(\mathrm{OR}=2,72)$, sibilos no início da vida $(\mathrm{OR}=2,79)$, eczema $(\mathrm{OR}=1,65)$, infecção respiratória no início da vida $(\mathrm{OR}=1,78)$ e rinite alérgica $(\mathrm{OR}=2,43)$ (Tabela 3 ). 
Análise não ajustada dos fatores associados à asma em escolares de 13 e 14 anos. São Luís, Maranhão, Brasil, 2009.

\begin{tabular}{|c|c|c|c|c|c|c|}
\hline \multirow[t]{3}{*}{ Variáveis } & \multicolumn{4}{|c|}{ Asma } & \multirow[t]{3}{*}{ OR (IC95\%) } & \multirow[t]{3}{*}{ Valor de $\mathrm{p}$} \\
\hline & \multicolumn{2}{|c|}{ Sim } & \multicolumn{2}{|c|}{ Não } & & \\
\hline & $\mathrm{n}$ & $\%$ & $\mathrm{n}$ & $\%$ & & \\
\hline Idade (anos) & & & & & & 0,708 \\
\hline 13 & 200 & 12,49 & 1.401 & 87,51 & $1,04(0,84-1,28)$ & \\
\hline 14 & 190 & 12,94 & 1.278 & 87,06 & 1,00 & \\
\hline Sexo & & & & & & 0,245 \\
\hline Feminino & 256 & 13,24 & 1.677 & 88,76 & $1,14(0,91-1,42)$ & \\
\hline Masculino & 134 & 11,80 & 1.002 & 88,20 & 1,00 & \\
\hline História familiar de asma & & & & & & $<0,001$ \\
\hline Sim & 119 & 30,36 & 273 & 69,64 & $3,86(3,01-4,96)$ & \\
\hline Não & 271 & 10,12 & 2.406 & 89,88 & 1,00 & \\
\hline Infecção respiratória no início da vida & & & & & & $<0,001$ \\
\hline Sim & 130 & 28,51 & 326 & 71,40 & $3,60(2,83-4,58)$ & \\
\hline Não & 260 & 9,95 & 2.353 & 90,05 & 1,00 & \\
\hline Nascimento pré-termo & & & & & & 0,013 \\
\hline Sim & 38 & 18,27 & 170 & 87,29 & $1,59(1,10-2,30)$ & \\
\hline Não & 352 & 12,30 & 2.509 & 87,70 & 1,00 & \\
\hline Exposição a tabaco & & & & & & $<0,001$ \\
\hline Sim & 154 & 16,85 & 760 & 83,15 & $1,64(1,32-2,05)$ & \\
\hline Não & 236 & 10,95 & 1.919 & 89,05 & 1,00 & \\
\hline Exposição a gato/cachorro & & & & & & 0,040 \\
\hline Sim & 269 & 13,63 & 1.705 & 86,37 & $1,26(1,01-1,59)$ & \\
\hline Não & 121 & 11,05 & 974 & 88,95 & 1,00 & \\
\hline Tabagismo materno na gravidez & & & & & & 0,021 \\
\hline $\operatorname{Sim}$ & 41 & 17,60 & 192 & 82,40 & $1,52(1,06-2,17)$ & \\
\hline Não & 349 & 12,31 & 2.487 & 87,69 & 1,00 & \\
\hline Sibilo no início da vida & & & & & & $<0,001$ \\
\hline Sim & 132 & 32,92 & 269 & 67,08 & $4,58(3,58-5,85)$ & \\
\hline Não & 258 & 9,67 & 2.410 & 90,33 & 1,00 & \\
\hline Eczema & & & & & & $<0,001$ \\
\hline Sim & 241 & 17,45 & 1.140 & 82,55 & $2,18(1,75-2,71)$ & \\
\hline Não & 149 & 8,83 & 1.539 & 91,17 & 1,00 & \\
\hline Rinite & & & & & & $<0,001$ \\
\hline Sim & 149 & 25,65 & 432 & 74,35 & $3,21(2,55-4,04)$ & \\
\hline Não & 241 & 9,69 & 2.247 & 90,31 & 1,00 & \\
\hline
\end{tabular}

IC95\%: intervalo de $95 \%$ de confiança; OR: odds ratio.

\section{Discussão}

No presente estudo, "sibilos nos últimos 12 meses" foram relatados por $12,7 \%$ dos escolares de 13 e 14 anos, valor próximo aos $13,8 \%$ encontrados por Felizola et al. 20 que avaliaram a prevalência de asma e sintomas relacionados no Distrito Federal, empregando o protocolo ISAAC em 3.254 adolescentes. A prevalência de asma medida por essa questão em nosso estudo também foi próxima à encontrada em Nova Iguaçu, que apontou prevalência de $11,7 \% 21$ e também próximo ao encontrado no estudo realizado por Breda et al. 22, nos municípios de Tubarão e Capivari de Baixo, que foi de $11,8 \%$. Em estudo realizado no Município de Duque de Caxias (Rio de Janeiro) com 4.040 estudantes de 13 e 14 anos com mesma metodologia, essa prevalência foi de $19 \% 13$ e em São Paulo, onde a metodologia ISAAC foi aplicada em 3.008 adolescentes, o valor encontrado foi $21,3 \% 10$. 
Análise multivariada dos fatores associados à asma em escolares de 13 e 14 anos. São Luís, Maranhão, Brasil, 2009.

\begin{tabular}{|c|c|c|}
\hline Variáveis & OR (IC95\%) & Valor de $p$ \\
\hline História familiar de asma & & $<0,001$ \\
\hline Não & 1,00 & \\
\hline Sim & $2,72(2,08-3,56)$ & \\
\hline Infecção respiratória no início da vida & & $<0,001$ \\
\hline Não & 1,00 & \\
\hline Sim & $1,78(1,33-2,39)$ & \\
\hline Eczema & & $<0,001$ \\
\hline Não & 1,00 & \\
\hline Sim & $1,65(1,31-2,09)$ & \\
\hline Exposição a tabaco & & 0,004 \\
\hline Não & 1,00 & \\
\hline Sim & $1,41(1,11-1,79)$ & \\
\hline Sibilos no início da vida & & $<0,001$ \\
\hline Não & 1,00 & \\
\hline Sim & $2,79(2,08-3,73)$ & \\
\hline Rinite alérgica & & $<0,001$ \\
\hline Não & 1,00 & \\
\hline Sim & $2,43(1,90-3,11)$ & \\
\hline
\end{tabular}

IC95\%: intervalo de 95\% de confiança; OR: odds ratio.

Na América Latina, segundo o ISAAC fase 1, a prevalência de asma ativa variou de $6,6 \%$ a $27 \%$ nos adolescentes, com os valores mais elevados de prevalência observados nos centros próximos à linha do Equador 23. No mundo, esta prevalência foi de 13,8\%, com variação de 1,6\% na Índia até $36,7 \%$ na Escócia 18 . Tais diferenças na prevalência entre os centros podem ser justificadas por fatores ambientais diversos. A baixa prevalência de asma encontrada em nosso estudo poderia ser justificada com a hipótese da higiene, onde a presença de atopia estaria relacionada inversamente à exposição a contaminantes orofecais, indicando que a estimulação nos primeiros anos de vida do trato gastrointestinal por agentes infecciosos seria importante na maturação do sistema imunológico da mucosa, reduzindo o risco de atopia 24 .

A questão "sibilos alguma vez na vida" do questionário do ISAAC é considerada pouco específica, porém com grande sensibilidade para o diagnóstico da asma, uma vez que várias doenças respiratórias podem cursar com sibilância. Entretanto, investigação de sibilos (chiado no peito) na faixa etária em estudo, em princípio, corresponde à asma, embora seja considerado um proxy para asma. Em nosso estudo, este relato esteve presente em $32,4 \%$ dos adolescentes, valor superior aos $29,5 \%$ observados em estudo realizado em Maceió, que também utilizou a metodologia ISAAC em 2.744 adolescentes 24 e abaixo da prevalência nacional, que variou de $35,4 \%$ a $46,9 \% 25$.

A avaliação da gravidade da asma pode ser obtida através das questões do estudo ISAAC relativas ao número de frequência de sibilos nos últimos 12 meses, ao sono prejudicado por sibilos nos últimos 12 meses e pela presença de dificuldade de fala devido a sibilos no último ano 26. Em nosso estudo, encontramos prevalência de $12,5 \%$ de adolescentes que relataram pelo menos uma crise de sibilo, de $8,5 \%$ que afirmaram ter tido sono prejudicado por sibilos e de $3,9 \%$ que tiveram dificuldade de fala por sibilos no último ano. Em Montes Claros (Minas Gerais), em estudo realizado por Maia et al. ${ }^{9} \mathrm{em}$ 3.770 adolescentes de 13 e 14 anos, estes resultados corresponderam respectivamente a $17,3 \%$, $11,8 \%$ e 3,2\%. Já na região oeste de São Paulo, em estudo da Fase III do ISAAC realizado por Pastorino 26 onde foram pesquisados 3.181 alunos também nessa faixa etária, os resultados foram $24,7 \%, 14,5 \%$ e 5,6, respectivamente. Resultados obtidos no Brasil pelo ISAAC - Fase I corresponderam a $21,5 \%, 11,5 \%$ e 4,9\%, respectivamente.

Nos países desenvolvidos há uma tendência de diminuição da gravidade das crises, com o emprego de terapia antiasmática eficaz 13 . A 
prevalência de asma grave nos estudantes de São Luís foi inferior às prevalências observadas em outros estudos realizados no país, como nos estudos realizados em Montes Claros por Maia et al. ${ }^{9}$ e na região oeste de São Paulo por Pastorino 26.

O diagnóstico de asma pela "presença de sibilos no último ano" tem se mostrado de maior sensibilidade para identificação dos possíveis asmáticos. Quando empregado o diagnóstico médico de asma pela questão "asma alguma vez na vida”, as taxas de prevalência de asma são muito baixas, como em Curitiba (8,6\%), Ribeirão Preto (9,7\%) e 9.9\% em Seropédica 27 .

Em nosso meio, é comum a população se referir à asma pelo nome de bronquite. Destaca-se, atualmente, que os termos asma e bronquite são equivocadamente utilizados como sinônimos por muitos médicos, dificultando o diagnóstico correto da asma ${ }^{13}$. Nos locais onde houve modificação do questionário padrão, tendo sido acrescentado o termo "bronquite", a prevalência de asma aumentou significativamente, como em Montes Claros (23,8\%) e em Cuiabá (Mato Grosso), que foi de $26,4 \% 27$. Sabe-se que a utilização do termo bronquite em inquérito epidemiológico sobre doença sibilante deve ser realizada com cautela, e não de forma isolada por apresentar baixa sensibilidade e especificidade para detecção de asma e correlação pobre com presença de sibilo e asma 28 . Em nosso estudo, a prevalência de "asma ou bronquite alguma vez na vida" foi de 19,1\%. A adição do termo "bronquite" no presente estudo determinou uma prevalência de as$\mathrm{ma} /$ bronquite maior que a prevalência do relato de sibilos nos 12 últimos meses. Essa modificação teve a intenção de aumentar a sensibilidade dessa questão e pode explicar parcialmente a prevalência mais elevada de resposta positiva encontrada. Em indivíduos na faixa etária infantil (entre 0 e 9 anos de idade) que participaram da PNAD 2003 que utilizou o diagnóstico médico como critério para o diagnostico de asma ou bronquite, houve uma prevalência média nacional de $8,1 \%$. Já na faixa etária acima dos 18 anos, esta prevalência foi de $12 \% 29$.

Quando perguntamos se houve "chiado no peito após exercícios físicos" e se houve "tosse seca noturna sem estar gripado ou com infecção respiratória" nos últimos doze meses, 9,8\% e 27\% responderam afirmativamente a essas questões, respectivamente. Tais resultados foram superiores aos encontrados pelo estudo ISAAC realizado em Itabira (Minas Gerais) 30, com prevalências iguais a $4,2 \%$ e $19,4 \%$ respectivamente, e inferiores aos encontrados pelo estudo ISAAC em Montes Claros 9, que foram de 16,6\% e 32,9\%, respectivamente. Esses dados tornam-se impor- tantes devido à tosse seca noturna ser manifestação importante da asma, principalmente na ausência de infecção das vias respiratórias 31 e a asma induzida pelo exercício poder permanecer como manifestação única da doença nessa faixa etária, nem sempre reconhecida pelos responsáveis e professores 21 . Vale ressaltar que a tosse seca noturna pode ser manifestação de refluxo gastroesofágico 32 e assim, com o emprego dessa questão tende-se a superestimar a prevalência de asma.

Ser do sexo masculino é um fator de risco para asma, como mostrado por Casagrande et al. 8 que, em estudo transversal integrante do ISAAC realizado na região oeste de São Paulo com 561 escolares de seis a sete anos, encontraram risco para asma 2,4 vezes maior para meninos. Parece que esta relação se inverte na adolescência, como apontado por Boechat et al. ${ }^{13}$ que, em estudo transversal realizado no município de Duque de Caxias, utilizando a metodologia do ISAAC em crianças de 6 e 7 anos e em adolescentes de 13 e 14 anos, encontraram prevalência maior de sibilos nos últimos doze meses entre os meninos na primeira faixa etária, com $\mathrm{RP}=1,17$, e maior entre as meninas no grupo de adolescentes, com $\mathrm{RP}=1,39$.

O fato de a prevalência de asma na infância ser maior entre os meninos está relacionado em parte ao menor diâmetro e maior tônus das vias aéreas e maiores níveis de IgE observados em meninos ${ }^{33}$. Na adolescência a prevalência fica maior para as meninas, o que parece ser decorrente, possivelmente, de fatores hormonais, além de que meninos têm a tendência de subestimar seus sintomas; ao contrário, as meninas podem superestimá-los 30. Em nossa casuística não houve diferença estatisticamente significante na prevalência de asma entre os sexos, resultado semelhante aos encontrados nos estudos realizados no Distrito Federal por Felizola et al. 20 e por Rosa et al. 34 em Tangará da Serra (Mato Grosso).

$\mathrm{Na}$ análise multivariada, foi observada associação positiva com história familiar de asma, infecção respiratória no início da vida, eczema, tabagismo passivo, sibilos no início da vida e rinite alérgica.

Com relação à associação com história familiar de asma, encontramos uma $\mathrm{OR}=2,72$. Em estudo realizado por Lintonjua et al. 35 foi demonstrado que a asma materna e paterna está relacionada à maior ocorrência da doença nas crianças maiores de cinco anos de idade, enquanto que nas crianças menores de cinco anos, relata-se associação apenas na presença de asma materna. Nas famílias em que um dos pais era asmático, as chances de ter uma criança com asma foram triplicadas. 
Infecções respiratórias no início da vida podem levar a episódios transitórios de sibilância na infância precoce. Em nosso estudo, sibilos e infecções respiratórias no início da vida apresentaram razões de prevalência de 2,79 e 1,78. Solé et al. 17 afirmaram que $50 \%$ a $65 \%$ das crianças asmáticas manifestaram seus primeiros sintomas durante o primeiro ano de vida.

Em estudo realizado por Vásquez et al. 36 foi observado que o tabagismo passivo foi um dos principais fatores de risco para o desenvolvimento de asma em adolescentes. Os resultados de nosso estudo mostraram que adolescentes expostos à fumaça de tabaco (tabagismo passivo) apresentaram prevalência de asma 1,41 vezes maior. Na atualidade, é praticamente consensual que o tabagismo passivo está associado a maiores prevalências de sibilos e asma, especialmente na primeira infância, com razão de chances de 1,2 a 1,39 .

É evidente a relação entre o surgimento precoce de eczema atópico e rinite antecedendo o desenvolvimento de asma, sendo tal fenômeno descrito pelo termo "marcha atópica" 37 . Os resultados do presente estudo identificaram nos adolescentes associação positiva entre eczema atópico e rinite com asma $(\mathrm{OR}=1,65$ e 2,43, respectivamente). Em estudo realizado na Alemanha foi demonstrado que, entre as 1314 crianças avaliadas, $50 \%$ das que apresentavam diagnóstico de eczema precoce e história familiar positiva apresentavam asma aos cinco anos de idade, enquanto apenas $12 \%$ das crianças sem esses diagnósticos, apresentavam asma ${ }^{38}$. Já em estudo realizado em Fortaleza (Ceará) em 3.015 adolescentes sobre associação de asma e rinite, foram evidenciadas elevadas taxas de asma e rinite, com significativa associação dos sintomas de rinite aos de asma e vice-versa, sugerindo que a presença concomitante da rinite aumenta significantemente a morbidade/gravidade da asma independentemente do envolvimento da atopia 39

Embora na análise univariada tabagismo materno durante a gravidez, exposição doméstica a animais peludos e nascimento pré-termo te- nham sido significantes, estas associações desapareceram na análise multivariada.

São conhecidos os efeitos do tabagismo dos pais sobre o sistema respiratório da criança. O tabagismo materno durante a gestação ou mesmo após o parto tem sido associado à manifestação de sibilância e asma 40. Entretanto em nosso estudo, esta associação não foi observada.

Animais domésticos peludos como cães e gatos podem causar ou agravar doenças respiratórias, como relatado por Brunekreef et al. 41 que mostraram ser a prevalência de asma diagnosticada por médico maior em crianças que possuíam gatos. Diferentemente disso, em nosso estudo, não encontramos associação entre exposição a cães e gatos e asma, resultado semelhante ao encontrado por Casagrande et al. ${ }^{8}$ que também não encontraram significância estatística quando pesquisaram tal associação.

Pereira 42, em estudo realizado sobre fatores de risco ambientais e familiares para sibilância em escolares da cidade de Uruguaiana (Rio Grande do Sul), observou que crianças nascidas pré-termo apresentaram maior frequência de doenças respiratórias, do que os nascidos a termo. Porém, Doyle 43 relatou melhora dos sintomas respiratórios e até mesmo da função pulmonar no decorrer da infância e da adolescência. Talvez por isso não se tenha observado em nosso estudo associação entre nascimento pré-termo e asma.

\section{Conclusão}

A prevalência de asma em adolescentes no presente estudo se encontra próxima à média mundial e abaixo da encontrada no Brasil, não havendo diferença significante entre os sexos. Dos sintomas de asma, apenas tosse seca noturna foi mais observada entre as meninas. Os fatores associados à asma identificados neste estudo foram história familiar de asma, infecções respiratórias no início da vida, eczema atópico, tabagismo passivo, sibilos no início da vida e rinite alérgica. 


\section{Resumo}

O objetivo deste estudo foi estimar a prevalência de asma e fatores associados em estudantes de 13 e 14 anos do Município de São Luís, Maranhão, Brasil. Realizouse estudo transversal utilizando o questionário do ISAAC (International Study of Asthma and Allergies in Childhood) e questionário complementar, entre julho de 2008 e maio de 2009 com 3.069 adolescentes. A prevalência de asma (sibilos nos últimos 12 meses) foi de 12,7\%, sendo que 32,4\% apresentaram sibilos alguma vez na vida, 3,9\% tinham dificuldade de fala por causa de sibilos e 9,8\% relataram sibilos após exercícios físicos. Na análise multivariada, os fatores associados à maior prevalência de asma foram história familiar de asma, infecção respiratória e sibilos no início da vida, eczema, tabagismo passivo e rinite alérgica. A prevalência de asma encontrada foi inferior à brasileira. Os fatores mais associados com a prevalência de asma foram história familiar de asma $(R P=3,86)$, sibilos no início da vida $(R P=4,58)$ e rinite alérgica $(R P=3,21)$.

Asma; Adolescente; Prevalência

\section{Colaboradores}

W. L. Lima, M. R. S. R. Costa e A. A. M. Silva contribuíram na concepção, análise e interpretação dos dados; na redação do artigo e revisão crítica relevante do conteúdo intelectual; e na aprovação final da versão a ser publicada. E. V. N. C. L. Lima e E. S. Costa colaboraram na concepção, na análise e interpretação dos resultados. A. M. Santos participou na concepção, análise e interpretação dos resultados, e na redação do artigo e revisão crítica do conteúdo intelectual.

\section{Referências}

1. Sociedade Brasileira de Pneumologia e Tisiologia/ Sociedade Brasileira de Alergia e Imunopatologia/ Sociedade Brasileira de Clínica Médica. IV Consenso Brasileiro no Manejo da Asma. J Bras Pneumol 2006; 32 Supl. 7:S447-74.

2. Global Initiative for Asthma. http://www.ginasth ma.org (acessado em 12/Abr/2009).

3. Masoli M, Fabian D, Holt S, Beasley R. The global burden of asthma: executive summary of the GINA dissemination committee report. Allergy 2004; 59:469-78.

4. Phelan PD. Asthma in children: epidemiology. BMJ 1994; 308:1584-5

5. Sears MR. Epidemiology of childhood asthma. Lancet 1997; 350:1015-20.

6. Fiore RW, Comparsi AB, Reck CL, Oliveira JK, Pampanelli KB, Fritscher CC. Variação na prevalência de asma e atopia em um grupo de escolares de Porto Alegre, Rio Grande do Sul. J Pneumol 2001; 27:237-42.
7. Arruda LK, Solé D, Baena-Cagnani CE, Naspitz CK. Risk factors for asthma and atopy. Curr Opin Allergy Clin Immunol 2005; 5:153-9.

8. Casagrande RRD, Pastorino AC, Souza RGL, Leone C, Solé D, Jacob CMA. Prevalência de asma e fatores de risco em escolares da cidade de São Paulo. Rev Saúde Pública 2008; 42:517-23.

9. Maia JGS, Marcopito LF, Amaral NA, Tavares BF, Santos FANL. Prevalência de asma e sintomas asmáticos em escolares de 13 e 14 anos de idade. Rev Saúde Pública 2004; 38:292-9.

10. Solé D, Yamada E, Vana A, Costa-Carvalho B, Naspitz C. Prevalence of asthma and related symptoms in school-age children in São Paulo, Brazil - International Study of Asthma and Allergies in Children (ISAAC). J Asthma 1999; 36:205-12.

11. Asher MI, Keil U, Anderson HR, Beasley R, Crane J, Manrtinez F, et al. International study of asthma and allergies in childhood (ISAAC): rationale and methods. Eur Respir J 1995; 8:483-91. 
12. Solé D, Wandalsen GF, Camelo-Nunes IC, Naspitz CK. Prevalência de sintomas de asma, rinite e eczema atópico entre crianças e adolescentes brasileiros identificados pelo International Study of Asthma and Allergies in Children (ISAAC) - fase 3. J Pediatr (Rio J.) 2006; 82:341-6.

13. Boechat JL, Rios JL, Sant'Anna CC, França AT. Prevalência e gravidade de sintomas relacionados à asma em escolares e adolescentes no município de Duque de Caxias, Rio de Janeiro. J Bras Pneumol 2005; 31:111-7.

14. Barros MBA, César CLG, Carandina L, Torre GD. Desigualdades sociais na prevalência de doenças crônicas no Brasil, PNAD 2003. Ciênc Saúde Coletiva 2006: 11:911-26.

15. International Study of Asthma and Allergies in Childhood Steering Committee. ISAAC phase one manual. 2nd Ed. Auckland/Munster: International Study of Asthma and Allergies in Childhood International Data Centre; 1993.

16. Ellwood P, Asher MI, Beasley R, Calyton TO, Stewart AW. ISAAC phase three manual. Auckland: International Study of Asthma and Allergies in Childhood International Data Centre; 2000.

17. Solé D, Vana A, Yamada E, Rizzo MCV, Naspitz CK. International Study of Asthma and Allergies in Childhood (ISAAC) written questionnaire: validation of the asthma component among Brazilian children. J Investig Allergol Clin Immunol 1998; 8:376-82.

18. International Study of Asthma and Allergies in Childhood Steering Committee. Worldwide variation in prevalence of symptoms of asthma, allergic rhinoconjunctivitis, and atopic eczema: ISAAC. Lancet 1998; 351:1225-32.

19. Reichenheim ME, Coutinho ES. Measures and models for causal inference in cross-sectional studies: arguments for the appropriateness of the prevalence odds ratio and related logistic regression. BMC Med Res Methodol 2010; 10:66.

20. Felizola MLBM, Veigas CAA, Almeida M, Ferreira F, Santos MCA. Prevalência de asma brônquica e de sintomas a ela relacionados em escolares do Distrito Federal e sua relação com o nível socioeconômico. J Bras Pneumol 2005; 31:486-91.

21. Kuschnir FC, Cunha AJLA, Braga DAC, Silveira HHN, Barroso MH, Aires ST. Asma em escolares de 13 e 14 anos do Município de Nova Iguaçu, Rio de Janeiro, Brasil: estimativas de prevalência, gravidade e diferenças de gênero. Cad Saúde Pública 2007; 23:919-26.

22. Breda D, Freitas PF, Pizzichini E, Agostinho FR, Pizzichini MMM. Prevalência de sintomas de asma e fatores de risco associados em adolescentes escolares de 13 e 14 anos dos municípios de Tubarão e Capivari de Baixo, Santa Catarina, Brasil. Cad Saúde Pública 2009; 25:2497-506.

23. Mallol J, Solé D, Asher I, Clayton T, Stein R, Soto Quiroz M, et al. Prevalence of asthma symptoms in Latin America: the international study of asthma and allergies in childhood (ISAAC). Pediatr Pulmonol 2000; 30:439-44.

24. Soares FJP, Santos MLM, Costa ADP, Andrade SMS, Medeiros MAS. Prevalência de asma brônquica em escolares e adolescentes do município de Maceió. Pediatria 2005; 27:95-102.
25. Costa SRR. Prevalência, gravidade e sintomas relacionados à asma em escolares de 6 até 8 anos e de 13 até 14 anos de Ribeirão Preto, SP, avaliados pelo ISAAC [Dissertação de Mestrado]. São Paulo: Faculdade de Medicina de Ribeirão Preto, Universidade de São Paulo; 2000.

26. Pastorino AC. Estudo da prevalência de asma e doenças alérgicas, da sensibilização a aeroalérgenos e da exposição a fatores de risco em escolares de 13-14 anos na região oeste da cidade de São Paulo [Dissertação de Mestrado]. São Paulo: Faculdade de Medicina, Universidade de São Paulo; 2005.

27. Solé D, Camelo-Nunes C. A dimensão do problema da asma e da rinite alérgica no Brasil: prevalência, hospitalizações e mortalidade. Gazeta Médica da Bahia 2008; 78:3-10.

28. Aires ST. Emprego dos termos asma ou bronquite em inquérito epidemiológico sobre doença sibilante entre escolares de Nova Iguaçu [Dissertação de Mestrado]. Rio de Janeiro: Universidade Federal do Rio de Janeiro; 2005.

29. Wehrmeister FC, Peres KGAP. Desigualdades regionais na prevalência de diagnóstico de asma em crianças: uma análise da Pesquisa Nacional por Amostra de Domicílios, 2003. Cad Saúde Pública 2010: 26:1839-52.

30. Solé D, Yamada E, Vana AT, Werneck G, Freitas LS, Sologuren MJ, et al. International study of asthma and allergies in childhood (ISAAC): prevalence of asthma and asthma-related symptoms among Brazilian schoolchildren. J Investig Allergol Clin Immunol 2001; 11:123-8.

31. Amorim AJ, Daneluzzi JC. Prevalência de asma em escolares. J Pediatr (Rio J.) 2001; 77:197-202.

32. Barbosa AB, Barbarena LS, Barbosa KLCP, Ribeiro DS. Manifestações laríngeas do refluxo laringofaríngeo e suas relações com hábitos alimentares manauenses. Arq Int Otorrinolaringol 2008; 12: 55-61.

33. Solé DA, Camelo Nunes IC, Rizzo MCV, Naspito CK. Asma na criança: classificação e tratamento. J Pediatr 1998; 74 Suppl 1:S48-58.

34. Rosa AM, Ignotti E, Hacon SS, Castro HAC. Prevalência de asma em escolares e adolescentes em um município na região da Amazônia brasileira. J Bras Pneumol 2009; 35:7-13.

35. Lintonjua AA, Carey VJ, Burge HA, Weiss ST, Gold DR. Parental history and the risk for childhood asthma. Does mother confer more risk than father? Am J Respir Crit Care Med 1998; 158:176-81.

36. Vásques NF, González AHS, Perales GM, Ochoa DL, Gómez MCB, Rodríguez EMV, et al. Association between family history of allergy, exposure to tobacco smoke, active smoking, obesity, and asthma in adolescents. Arch Bronconeumol 2006; 42:621-6.

37. Spergel JM. Atopic march: link to upper airways. Curr Opin Allergy Clin Immunol 2005; 5:17-21.

38. Lau S, Illi S, Sommerfeld C, Niggemann B, Bergmann R, von Mutius E, et al. Early exposure to house-dust mite and cat allergens ans development of childhood asthma: a cohort study. Multicentre Allergy Study Group. Lancet 2000; 356:1392-7.

39. Luna MFG, Almeida PC, Silva MGC. Prevalência e associação de asma e rinite em adolescentes de 13 e 14 anos de Fortaleza, Ceará, Brasil. Cad Saúde Pública 2011; 27:103-12. 
40. Carlsen KH, Lodrup CKC. Parental smoking and childhood asthma: clinical implications. Treat Respir Med 2005; 4:337-46.

41. Brunekreef B, Groot, B, Hoek G. Pets, allergy and respiratory symptoms in children. Int J Epidemiol 1992; 21:338-42.

42. Pereira MU. A relação de fatores de risco ambientais e familiares com sibilância em escolares da cidade de Uruguaiana, RS [Dissertação de Mestrado]. Porto Alegre: Faculdade de Medicina, Pontifícia Universidade Católica do Rio Grande do Sul; 2005.
43. Doyle LW. Growth and respiratory health in adolescence of the extremely low-birth weight survivor. Clin Perinatol 2000; 27:421-32.

Recebido em 11/Jan/2011

Versão final reapresentada em 02/Mar/2012

Aprovado em 19/Mar/2012 